\title{
CONTAMINAÇÃO POR METAIS PESADOS EM ÁREAS AGRÍCOLAS NO ESTADO DO TOCANTINS
}

\author{
Michele Ribeiro Ramos ${ }^{l}$, Mônica Ferreira De Abreu², João Vitor De Medeiros Guizzo ${ }^{1}$, Divonzil Gonçalves Cordeiro ${ }^{4}$, Phillipe Farias \\ Ferreira ${ }^{5}$
}

\begin{abstract}
${ }^{\text {I} E n g e n h a r i a ~ A g r o n o ̂ m i c a, ~ U n i v e r s i d a d e ~ E s t a d u a l ~ d o ~ T o c a n t i n s, ~ 77000-000, ~ P a l m a s, ~ T O, ~ B r a s i l ; ~}{ }^{2}$ Centro de Solos e Recursos Agroambientais, Instituto Agronômico de Campinas, 13075-630, Campinas, SP, Brasil; ${ }^{4}$ Núcleo Temático de Sistemas Agrícolas Integrados, Embrapa Pesca e Aquicultura, Palmas, TO; ${ }^{5}$ Instituto Federal de Educação, Ciência e Tecnologia do Tocantins, 77000 000, Palmas, TO, Brasil.
\end{abstract}

*E-mail: michele.rr@unitins.br

\section{RESUMO}

O presente trabalho consistiu na coleta de amostras de solos para análise e descrição dos valores de metais pesados em áreas agrícolas de alguns municípios do estado do Tocantins. O objetivo é fazer comparações com os Valores de Referência estabelecidos para os estados de São Paulo (SP) e Minas Gerais (MG), uma vez que não existem valores de referência para o estado do Tocantins. Amostras de solo foram coletadas em 27 áreas agrícolas e submetidas ao método de digestão EPA3051, proposto pela Agência de Proteção Ambiental (EPA). As amostras de solos apresentaram diferentes níveis de concentração de metais pesados, com arsênico (As), mercúrio (Hg) e selênio (Se) sendo mais constantes. Não houve níveis de metais pesados próximos aos Valores da Pesquisa Agrícola, apenas alguns elementos excederam o limite dos Valores de Prevenção e os Valores de Referência da Qualidade (QRV) propostos para os estados de SP, bem como para o QRV estabelecido para as áreas no estado de MG. Essas concentrações, no entanto, não apresentam risco de contaminação do solo.

Palavras-chave: CONAMA 420. Referência de qualidade. Impacto ambiental. Legislação.

\section{Introdução}

A legislação ambiental brasileira é composta por um conjunto de leis, decretos e medidas provisórias, federais, estaduais e municipais, dentre as quais se destacam a Política Nacional de Meio Ambiente (Lei 6.938/81), a Política de Recursos Hídricos (Lei Estadual 7.663/91 e Lei Federal 9.433/97), e a Lei de Proteção da Vegetação Nativa (Lei 12.651/2012), e que abordam as restrições ao uso e ocupação dos solos agrícola e urbano [1].

Cobbina et al. [2] definem metais pesados como um grupo de compostos químicos ubíquos e não-biodegradáveis. Quando em altas concentrações, exercem efeitos tóxicos sobre animais e plantas, embora alguns desses metais podem ser benéficos, quando em concentrações baixíssimas [3].

A fim de parametrizar os limites desta toxidez, a Resolução CONAMA No20, de 28 de dezembro de 2009, dispõe sobre critérios e valores orientadores de qualidade do solo (valores de prevenção e valores de investigação) quanto à presença de substâncias químicas. Estabelece, também, diretrizes para o gerenciamento ambiental de solos contaminados por esses compostos químicos em decorrência de atividades antrópicas. Os critérios contidos nesta resolução são similares aos critérios praticados atualmente nos países desenvolvidos, incorporando a avaliação de risco e o uso pretendido do solo [4].

$\mathrm{O}$ primeiro dos valores referenciais desta normativa constitui o valor de referência de qualidade (VRQ), que representa a medida da concentração natural de elementos químicos em solos sem influência humana [5].

Este parâmetro é, portanto, a base fundamental para o estabelecimento do valor máximo permitido de um determinado metal nos solos de uma região. Presta-se como instrumento para a geração e implantação de políticas públicas visando ao uso e ao manejo do solo, e ainda serve como base de referência para os órgãos ambientais estaduais monitorarem os níveis de contaminação dos solos.

A partir do VRQ, é possível estimar os dois outros parâmetros contidos da resolução: o valor de prevenção (VP) e o valor de investigação (VI). O primeiro deles (VP) é definido como a concentração de valor limite de determinada substância 
no solo, tal que ele seja capaz de sustentar as suas funções principais. Em outras palavras, este valor indica um ponto a partir do qual as funções primárias do solo ficarão comprometidas, tanto no que diz respeito à biota dos solos, quanto à qualidade das águas subterrâneas, observada as consequências sobre a saúde humana [6].

Por sua vez, o valor de investigação (VI) pode ser compreendido como a concentração de determinada substância no solo ou na água subterrânea acima da qual existem riscos potenciais, diretos ou indiretos, à saúde humana, considerando um cenário de exposição padronizado [1]. Quando os valores de metais pesados de um determinado solo ultrapassam os valores de investigação (VI), faz-se necessário o emprego de medidas para eliminar o excesso de metais do solo contaminado.

Embora o estado do Tocantins não possua nenhuma estimativa de nenhum destes valores, o objetivo deste trabalho é diagnosticar os teores dos metais pesados arsênio (As), cádmio $(\mathrm{Cd})$, chumbo $(\mathrm{Pb})$, cromo $(\mathrm{Cr})$, mercúrio $(\mathrm{Hg})$, níquel $(\mathrm{Ni})$ e selênio ( $\mathrm{Se}$ ) em solos de áreas agrícolas e inferir sobre os níveis de contaminação de solos dos municípios da região central do estado do Tocantins.

\section{Metodologia}

\section{1 Área do estudo}

Inicialmente foram realizadas viagens em alguns municípios de grande potencial agrícola, a fim de selecionar áreas para a coleta de amostras. As áreas escolhidas estão situadas na região central do estado do Tocantins (Figura 1), nos municípios de Pedro Afonso ( $8^{\circ} 58^{\prime} 42^{\prime \prime} \mathrm{S} ; 48^{\circ} 10^{\prime} 23^{\prime \prime} \mathrm{W}$ ),

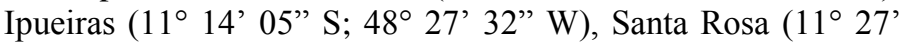
00" S; 47 07' 09" W), Monte do Carmo (10 45' 52" S; $48^{\circ}$ 06' 41” W) e Porto Nacional ( $10^{\circ} 42$ ' 33” S; $48^{\circ} 24$ ' 14” W).

As coletas das amostras foram realizadas nos dois principais solos do estado do Tocantins (em área e de maior importância agrícola), quais sejam, LATOSSOLOS VERMELHO-AMARELO e PLINTOSSOLOS PÉTRICOS CONCRECIONÁRIOS [7]. Nos locais escolhido, foram realizadas diversas coletas de amostras de solos, sendo oito (8) propriedades em Pedro Afonso, uma (1) em Ipueiras, onze (11) em Santa Rosa, cinco (5) em Monte do Carmo e duas (2) em Porto Nacional, totalizando 27 amostragens. Priorizando as culturas do milho, soja, feijão e cana-de-açúcar.

Através de caminhadas aleatórias nas áreas escolhidas, foram coletadas múltiplas amostras simples, somente na camada de superfície do solo $(0-20 \mathrm{~cm})$. Estas amostras simples foram, posteriormente, misturadas para compor uma amostra composta. Estas foram armazenadas em sacolas plásticas esterilizadas e encaminhadas para análise em laboratório.

\subsection{Análises químicas e granulométricas}

As amostras foram levadas para o Laboratório Agroambiental, do Centro de Ciências de Agrárias, da Universidade Estadual do Tocantins, onde foram secas ao ar e, posteriormente, passadas em peneiras de $2 \mathrm{~mm}$, para a segregação da terra fina.

Posteriormente, foram realizadas as análises químicas ( $\mathrm{Ca}, \mathrm{Mg}, \mathrm{K}, \mathrm{P}, \mathrm{pH}$ em água) e de granulometria (areia, silte e argila). $\mathrm{O}$ pH do solo, a acidez trocável (Al), os teores de $\mathrm{P}, \mathrm{K}$, $\mathrm{Ca}$ e $\mathrm{Mg}$ trocáveis, acidez potencial $(\mathrm{H}+\mathrm{Al})$, matéria orgânica saturada (M.O.S) e carbono orgânico (C) foram determinadas de acordo com metodologia apresentada pela EMBRAPA [8]. A partir dos valores de acidez potencial, bases trocáveis e alumínio trocável, foram calculados a soma de bases (SB), a capacidade de troca de cátions (CTC), a porcentagem de saturação por bases $(\mathrm{V} \%)$ e a porcentagem de saturação por alumínio $(\mathrm{m} \%)$.

\subsection{Análises de metais pesados}

As amostras de solos foram colocadas dentro de béqueres de $50 \mathrm{~mL}$, onde foram secos em estufa a $150^{\circ} \mathrm{C}$, durante 24 horas. Em seguida, aproximadamente $10 \mathrm{~g}$ do material seco foi colocado em saquinhos plásticos que foram adequadamente vedados e identificados.

Posteriormente, estas amostras foram encaminhadas para o Centro de Solos e Recursos Agroambientais, no Laboratório de Análise de Solo e Planta do Instituto Agronômico de Campinas (IAC), no estado de São Paulo, onde foi feita a determinação dos teores totais de metais pesados.

Foram determinados os teores totais de arsênio (As), cádmio $(\mathrm{Cd})$, chumbo $(\mathrm{Pb})$, cromo $(\mathrm{Cr})$, mercúrio $(\mathrm{Hg})$, níquel (Ni) e selênio ( $\mathrm{Se}$ ). As amostras de solo foram submetidas ao método de digestão (EPA3051 da Environmental Protection Agency - EPA/EUA) [9], o qual é recomendado pelo Conselho Nacional do Meio Ambiente [1]. Cerca de $300 \mathrm{mg}$ de amostra foram pesados diretamente nos frascos de PFA (perfluoroalcóxi - rosqueáveis de baixa pressão) onde adicionou-se $6,00 \mathrm{~mL}$ de $\mathrm{HNO}_{3} 65 \%(\mathrm{v} / \mathrm{v})$ e $2,00 \mathrm{~mL}$ de $\mathrm{HCl} 37 \%$ (v/v). A digestão foi efetuada em forno de micro-ondas fechado, com cavidade, por 5 minutos e 30 segundos na rampa de temperatura, tempo necessário para atingir $175^{\circ} \mathrm{C}$, mantendo-se essa temperatura por mais 4 minutos e 30 segundos. Após resfriamento, as amostras foram transferidas para frascos de polietileno e o volume ajustado para $15 \mathrm{~mL}$ com água ultrapura. Os extratos foram obtidos após centrifugação por 15 minutos a $2500 \mathrm{rpm}$. As digestões foram realizadas em triplicatas. 
As medidas dos elementos foram determinadas por espectrometria de emissão óptica em plasma indutivamente acoplado (ICP-OES).

\section{Resultados e discussões}

Como o estado do Tocantins ainda não possui nenhum dado para os valores orientadores de contaminação por metais pesados (Valores de Referência de Qualidade - VRQ), para fins comparativos, foram utilizados os valores orientadores de algumas substâncias segundo as normativas publicadas para solos $\left(\mathrm{mg} \mathrm{kg}^{-1}\right)$ do estado de São Paulo [10] e do estado de Minas Gerais [11].

Tabela 1 - Valores orientadores, em $\mathrm{mg} \mathrm{kg}^{-1}$, estabelecidos pela CETESB [10].

\begin{tabular}{cccc} 
Elementos & $\begin{array}{c}\text { Valor de Referência } \\
\text { de Qualidade }\end{array}$ & $\begin{array}{c}\text { Valor de } \\
\text { Prevenção }\end{array}$ & $\begin{array}{c}\text { Valor de } \\
\text { Intervenção } \\
\text { Agrícola }\end{array}$ \\
\hline Arsêncio & 3,5 & 15 & 35 \\
Cádmio & $<0,5$ & 1,3 & 3,6 \\
Chumbo & 17 & 72 & 150 \\
Cromo & 40 & 75 & 150 \\
Mercúrio & 0,05 & 0,5 & 1,2 \\
Níquel & 13 & 30 & 190 \\
Selênio & 0,25 & 1,2 & 24 \\
\hline
\end{tabular}

Fonte: Extraído, CETESB

Tabela 2 - Valores orientadores, em $\mathrm{mg} \mathrm{kg}^{-1}$, estabelecidos pelo COPAM [11].

\begin{tabular}{cccc}
\hline Elementos & $\begin{array}{c}\text { Valor de } \\
\text { Referência de } \\
\text { Qualidade }\end{array}$ & $\begin{array}{c}\text { Valor de } \\
\text { Prevenção }\end{array}$ & $\begin{array}{c}\text { Valor de } \\
\text { Intervenção } \\
\text { Agrícola }\end{array}$ \\
\hline Arsêncio & 8 & 15 & 35 \\
Cádmio & $<0,4$ & 1,3 & 3 \\
Chumbo & 19,5 & 72 & 180 \\
Cromo & 75 & 75 & 150 \\
Mercúrio & 0,05 & 0,5 & 12 \\
Níquel & 21,5 & 30 & 70 \\
Selênio & 0,5 & 5 & $\ldots$ \\
\hline
\end{tabular}

Fonte: Extraído COPAM

\subsection{Parâmetros de fertilidade dos solos investigados}

A distribuição das diferentes formas de metais depende das propriedades químicas dos metais e das características do solo, tais como: $\mathrm{pH}$, textura, capacidade de troca catiônica (CTC), matéria orgânica [3]. $\mathrm{O}$ pH afeta a distribuição química, mobilidade e disponibilidade de elementos [12]. A CTC é um dos principais fatores que influenciam na disponibilidade de metais pesados no solo, sendo que, quanto maior a CTC, mais sítios de adsorção estarão disponíveis nos coloides do solo para adsorver metais [13].

Nas áreas cultivadas com soja, somente duas delas apresentaram valores de CTC maiores do que $15 \mathrm{cmol} \mathrm{dm}^{-3}$ (Tabela 3). Ambas estão situadas no município de Pedro Afonso, embora pertençam a duas classes de solos distintas (PLINTOSSOLO PÉTRICO Concrecionário 1 e LATOSSOLO VERMELHO AMARELO Distrófico 2). Isso significa que esses dois tipos de solos disponibilizam uma quantidade maior de sítios de ligação, podendo facilitar a adsorção de metais pesados. O PLINTOSSOLO PETRICO Concrecionário 4 (Santa Rosa) e o LATOSSOLO VERMELHO AMARELO Distrófico 10 (Porto Nacional), apresentaram os menores valores de CTC, o que lhes confere menor capacidade da adsorção de metais.

De acordo com a análise química, o PLINTOSSOLO PÉTRICO Concrecionário 1 (Pedro Afonso), o LATOSSOLO VERMELHO AMARELO Distrófico 2 (Pedro Afonso) e o PLINTOSSOLO PETRICO Concrecionário 2 (Santa Rosa), apresentaram os maiores teores de matéria orgânica do solo (MOS), atingindo valores de $4,2 \%, 4,5 \%$ e $3,0 \%$, respectivamente. Esses valores são considerados altos [14] (Tabela 3), o que pode definir maior poder de complexação de metais. De fato, a presença de grupamentos funcionais da MOS pode atuar complexando metais presentes na solução do solo e, desta forma, diminuir a toxicidade desses poluentes [15].

Em condições de pH acima de 6 (seis), como é o caso dos LATOSSOLOS VERMELHO AMARELO Distrófico 6 e 7 (Monte do Carmo) (Tabela 3), é favorecida a dissociação de $\mathrm{H}^{+}$ a partir de grupos $\mathrm{OH}$ da matéria orgânica, bem como dos óxidos de $\mathrm{Fe}$ e $\mathrm{Al}$. Isso leva ao aumento da adsorção dos metais e posterior precipitação [16], afetando assim sua biodisponibilidade.

O LATOSSOLO VERMELHO AMARELO Distrófico 7 (Monte do Carmo) apresentou V\% de 58,6, o maior percentual desse parâmetro verificado entre todos os solos coletados, caracterizando-o como eutrófico. Em contrapartida, o PLINTOSSOLO PÉTRICO Concrecionário 4 (Santa Rosa) apresentou a menor porcentagem de V\% (Tabela 3). 
Tabela 3 - Análise química e física dos solos cultivados com soja.

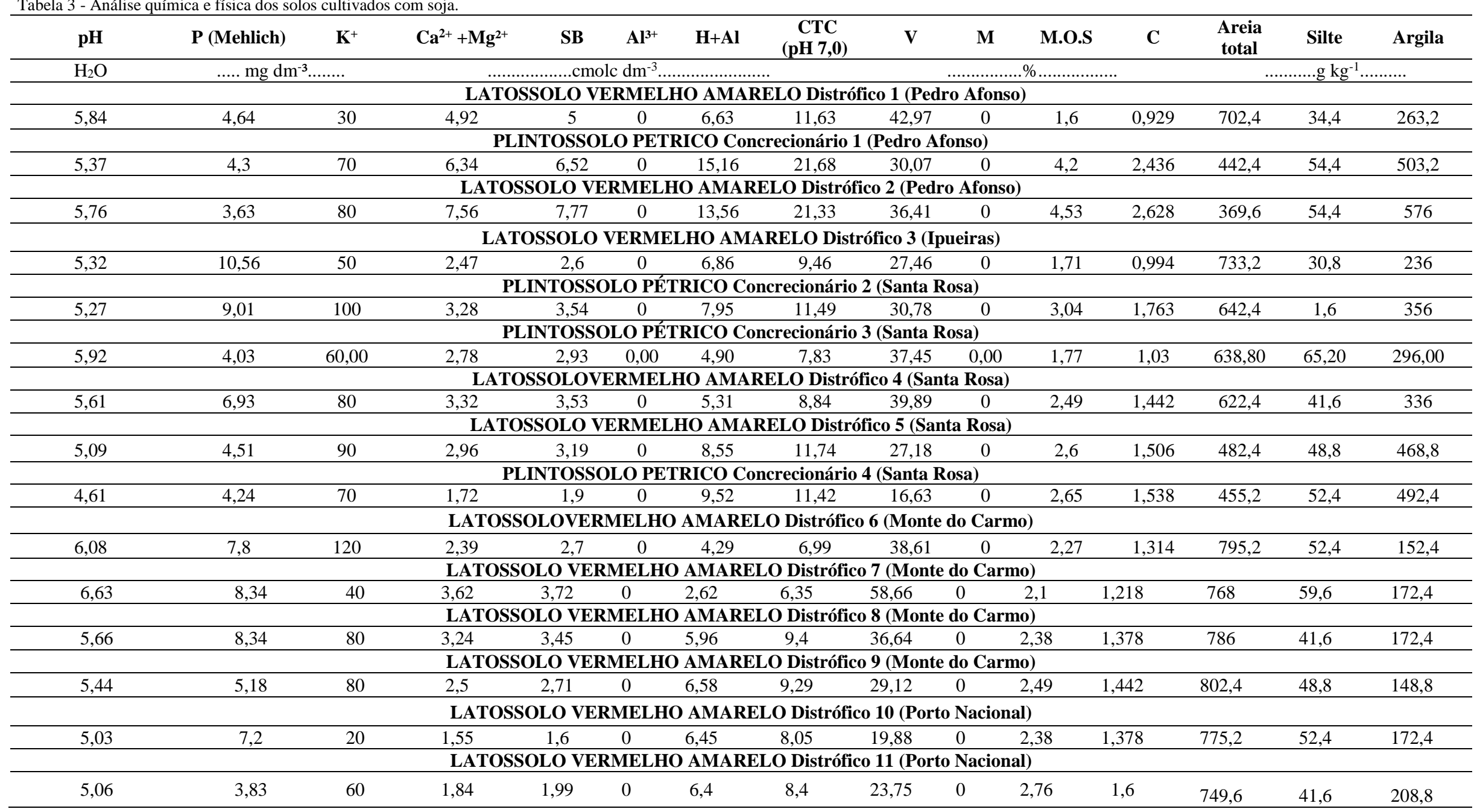


A textura do solo é definida pela proporção relativa de cada uma das classes de tamanho de partículas de um solo. De acordo com a análise granulométrica, nota-se que, dentre as amostras, há grande amplitude de classes texturais.

Como os teores de argila dos situados entre $350 \mathrm{~g} \mathrm{~kg}^{-1}$ e $600 \mathrm{~g} \mathrm{~kg}^{-1}$, os PLINTOSSOLOS PETRICO Concrecionário1, 2, e 4 (Pedro Afonso; Santa Rosa; Santa Rosa) e os LATOSSOLOS VERMELHO AMARELO Distrófico 2 e 5 (Pedro Afonso; Santa Rosa), são solos de textura argilosa (Tabela 3). Por sua vez, como os LATOSSOLOS VERMELHO AMARELO Distrófico 1, 3, 4 e 11 (Pedro Afonso; Ipueiras; Santa Rosa, Porto Nacional) e o PLINTOSSOLO PÉTRICO Concrecionário 3 (Santa Rosa) enquadram-se em solos de textura média-argilosa, por possuírem teores de argila variando entre 200 - $350 \mathrm{~g} \mathrm{~kg}^{-1}$, além de teores de areia situados acima de $600 \mathrm{~g} \mathrm{~kg}^{-1}$.

Já os LATOSSOLOS VERMELHO AMARELO Distrófico 6, 7, 8, 9 e 10 (Monte do Carmo; Monte do Carmo; Monte do Carmo; Monte do Carmo; Porto Nacional) apresentam textura média-arenosa, com teores de areia acima de $750 \mathrm{~g}$ $\mathrm{kg}^{-1}$ e menos de $200 \mathrm{~g} \mathrm{~kg}^{-1}$ de argila (Tabela 3) [7]. Sob o ponto de vista prático, espera-se que os solos argilosos tenham maior capacidade de retenção por adsorção dos metais. Ao mesmo tempo, solos arenosos devem estar mais prontamente sujeitos à contaminação, diante de sua baixa capacidade adsortiva [17], providenciando condições para a lixívia de metais solúveis em profundidade.

Nos locais de coleta onde os solos encontravam-se sob o cultivo do milho (Tabela 4), os PLINTOSSOLOS PÉTRICOS Concrecionário 1, 2, e 3 (Pedro Afonso) e os LATOSSOLOS VERMELHO AMARELO Distrófico 1 (Pedro Afonso) e 4 (Santa Rosa) apresentam valores de CTC maiores que 15 cmol dm $\mathrm{dm}^{-3}$ (Tabela 4), o que permite enquadrá-los como solos de alta capacidade de troca de cátions [14]. Isso lhes atribui a propriedade de adsorver maior quantidade de metais devido à uma maior quantidade de sítios de ligação ou, cargas negativas. Em relação aos valores de MOS, destacam-se novamente os PLINTOSSOLOS PÉTRICOS
Concrecionário 1, 2 e 3 (Pedro Afonso; Santa Rosa; Santa Rosa) e os LATOSSOLOS VERMELHO AMARELHO Distrófico 1 e 4 (Pedro Afonso; Santa Rosa), com percentual de MOS acima de 3\% (Tabela 4) [14]). Portanto, além de apresentarem boa capacidade de trocar cátions, também apresentam teores de matéria orgânica adequados à filtragem de metais presentes no solo, em função de sua configuração química e profusão de grupos fenólicos e carboxílicos [18].

Nas áreas cultivadas com milho, em nenhuma amostra de solo foi verificado $\mathrm{pH}$ acima de 6 . Este parâmetro variou entre 4,69 e 5,68 (Tabela 4), indicando acidez média $(5,0$ - 5,9) e acidez elevada $(<5,0)$ [7]. Para o bom desenvolvimento da cultura, o pH ideal deveria se situar na faixa de 6 a 7,2. Do ponto de vista de sua capacidade adsortiva, os valores de $\mathrm{pH}$ encontrados representam pontos de alta e média capacidade de solubilidade de metais no solo, visto que, os metais pesados são mais móveis em condições de solos mais ácidos [3].

Todos os solos investigados apresentaram caráter distrófico, com valor V\% bem inferior a $50 \%$ [14]. A saturação por bases foi baixa para estes solos, sendo o maior valor verificado no PLINTOSSOLO PÉTRICO Concrecionário 2 (Pedro Afonso).

Os teores de argila oscilaram entre 350 - 600 $\mathrm{g} \mathrm{kg}^{-1}$ (Tabela 4), o que caracteriza solos de textura argilosa, ou seja, bem estruturados e como uma boa capacidade de adsorver cátions e íons, sendo considerados áreas de boa qualidade para cultivo do milho, [7]. Entretanto, o LATOSSOLO VERMELHO AMARELO Distrófico 1 (Pedro Afonso) se destacou por apresentar teor de argila pouco acima de $600 \mathrm{~g} \mathrm{~kg}^{-1}$ (Tabela 4), o que permite enquadrá-lo como um solo de textura muito argilosa, consequentemente, de excelente estrutura, com alta capacidade de retenção de água, devido a sua CTC mais elevado, o que lhe confere menor perda de nutrientes por lixiviação, sendo além disso, mais firme e resistente à erosão [7]. Dadas tais características, é esperado que tenha maior capacidade reter metais pesados. 
Tabela 4 - Análise química e física dos solos cultivados com milho.

\begin{tabular}{|c|c|c|c|c|c|c|c|c|c|c|c|c|c|c|}
\hline pH & P (Mehlich) & $\mathbf{K}^{+}$ & $\mathrm{Ca}^{2+}+\mathrm{Mg}^{2+}$ & SB & $\mathbf{A l}^{3+}$ & $\mathbf{H}+\mathbf{A l}$ & CTC $(\mathbf{p H} 7,0)$ & $\mathbf{V}$ & $\mathbf{m}$ & M.O.S & $\mathbf{C}$ & Areia total & Silte & Argila \\
\hline $\mathrm{H}_{2} \mathrm{O}$ & \multicolumn{2}{|c|}{......mg dm³......... } & \multicolumn{9}{|c|}{ 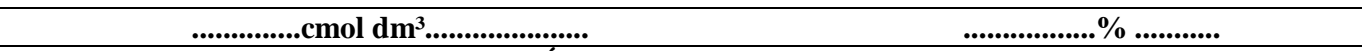 } & \multicolumn{3}{|c|}{.............. k kg-1................. } \\
\hline \multicolumn{15}{|c|}{ PLINTOSSOLO PÉTRICO Concrecionário 1 (Pedro Afonso) } \\
\hline 5,1 & 6,99 & 60 & 5,92 & 6,07 & 0 & 15,92 & 22 & 27,61 & 0 & 4,92 & 2,852 & 462,4 & 41,6 & 496 \\
\hline \multicolumn{15}{|c|}{ PLINTOSSOLO PÉTRICO Concrecionário 2 (Pedro Afonso) } \\
\hline 5,68 & 6,66 & 120 & 8,19 & 8,5 & 0 & 12,89 & 21,38 & 39,74 & 0 & 4,25 & 2,468 & 422,4 & 94,4 & 483,2 \\
\hline \multicolumn{15}{|c|}{ PLINTOSSOLO PÉTRICO Concrecionário 3 (Pedro Afonso) } \\
\hline 5,22 & 12,78 & 110 & 6,53 & 6,81 & 0 & 16,19 & 23 & 29,62 & 0 & 3,32 & 1,923 & 542,4 & 54,4 & 403,2 \\
\hline \multicolumn{15}{|c|}{ LATOSSOLO VERMELHO AMARELO Distrófico 1 (Pedro Afonso) } \\
\hline 5,11 & 6,39 & 120 & 4,94 & 5,25 & 0 & 17,9 & 23,15 & 22,67 & 0 & 4,86 & 2,82 & 302,4 & 81,6 & 616 \\
\hline \multicolumn{15}{|c|}{ LATOSSOLO VERMELHO AMARELO Distrófico 2 (Santa Rosa) } \\
\hline 5,47 & 5,04 & 70 & 2,88 & 3,06 & 0 & 8,99 & 12,05 & 25,39 & 0 & 1,88 & 1,09 & 442,4 & 41,6 & 516 \\
\hline \multicolumn{15}{|c|}{ LATOSSOLO VERMELHO AMARELO Distrófico 3 (Santa Rosa) } \\
\hline 5,54 & 6,46 & 120 & 4,28 & 4,59 & 0 & 9,08 & 13,66 & 33,58 & 0 & 2,65 & 1,538 & 478,8 & 45,2 & 476 \\
\hline \multicolumn{15}{|c|}{ PLINTOSSOLO PETRICO Concrecionário 4 (Santa Rosa) } \\
\hline 4,69 & 4,64 & 80 & 2,25 & 2,46 & 0 & 12,34 & 14,8 & 16,59 & 0 & 2,27 & 1,31 & 415,2 & 48,8 & 536 \\
\hline \multicolumn{15}{|c|}{ LATOSSOLO VERMELHO AMARELO Distrófico 4 (Monte do Carmo) } \\
\hline 5,05 & 8,34 & 100 & 3,96 & 4,22 & 0 & 12,38 & 16,59 & 25,41 & 0 & 3,09 & 1,795 & 309,6 & 101,6 & 588,8 \\
\hline
\end{tabular}


Sob o cultivo da cana-de-açúcar (Tabela 5), os dois LATOSSOLOS VERMELHO AMARELO Distróficos cujas amostras foram submetidas à análise apresentaram valores médios de CTC, entre 5 - $15 \mathrm{cmol} \mathrm{dm}^{-3}$ [14] (Tabela 5). Logo, apresentam uma capacidade mediana de troca de cátions, tornando-os aptos à adsorção de metais.

Os níveis de $\mathrm{pH}$ encontrados foram baixo $(<$ 5,0 - acidez elevada) e médio (5,0 - 5,9 - acidez média) [14]. Por outro lado, o aumento do $\mathrm{pH}$ no solo poderá elevar os níveis de CTC, além de favorecer a formação de complexos e quelatos, mediante aumento dos teores de matéria orgânica, fato que minimiza a disponibilidade dos metais pesados às plantas e diminui sua mobilidade no solo [19]. Em relação a porcentagem de MOS, os dois LATOSSOLOS VERMELHO AMARELO Distróficos apresentaram teores médios deste descritor no solo, variando entre 1,5 a 3\% (Tabela 5) [14].

Nas duas amostras, a estimativa da porcentagem da saturação por base (V\%) estava abaixo de $50 \%$ (Tabela 5), o que lhe confere o nome distrófico [14]. A despeito disso, o LATOSSOLO VERMELHO AMARELO Distrófico 2 (Pedro Afonso) se destacou por apresentar textura argilosa e grande potencial agrícola, com teor de argila entre $350 \mathrm{~g} \mathrm{~kg}^{-1}$ e $600 \mathrm{~g} \mathrm{~kg}^{-1}$ (Tabela 5), característica esta também verificada para o LATOSSOLO VERMELHO AMARELO Distrófico 1 (Pedro Afonso), mesmo diante de uma textura médiaargilosa. Portanto, o primeiro solo indica uma maior capacidade de adsorver os metais em sua composição em relação ao segundo diminuindo a chance de solubilidade.

Já em relação aos níveis de V\%, os dois solos apresentaram valores inferiores a $50 \%$, por isso, distróficos e com baixo nível de cargas negativas ocupadas por cátions básicos (Tabela 6) [14]. Entretanto, valores acima de $40 \%$, como aquele verificado para o LATOSSOLO VERMELHO AMARELO, ora em análise, já é tido como ótimo para a prática de agricultura nos solos do cerrado.

Quanto à presença de MOS, os dois solos apresentaram níveis médios de concentração, entre 1,5 - 3\% (Tabela 6) [14]. Ainda nas áreas cultivadas por feijão, o PLINTOSSOLO PÉTRICO Concrecionário (Pedro Afonso) apresentou maior teor de argila em sua composição, com 556 g. $\mathrm{kg}^{-1}$ (Tabela 6), caracterizando um solo de textura argilosa, enquanto o LATOSSOLO VERMELHO AMARELO Distrófico (Pedro Afonso) se destacou pelo elevado teor de areia, com 769,6 g. $\mathrm{kg}^{-1}$, associado a teores de argila abaixo de 200 g. $\mathrm{kg}^{-1}$ (Tabela 6). É esperado, portanto, que apresentem mais facilidade de solubilização de metais devido a sua baixa capacidade de adsorção.

\subsection{Metais pesados no solo}

\subsubsection{Teores de metais pesados em solos cultivados com soja}

No solo sob cultivo da soja os teores de arsênio (As) variaram de $<0,1 \mathrm{mg} \cdot \mathrm{kg}^{-1}$ a $10,4 \mathrm{mg} \cdot \mathrm{kg}^{-}$ ${ }^{1}$. Se usados os critérios para o estado de São Paulo [10], onde o VRQ é igual a 3,5 mg. $\mathrm{kg}^{-1}$ (Tabela 1), parte dos solos pesquisados estão acima do estabelecido, ou seja, os valores estão acima dos teores naturais nessas áreas. Esse resultado pode indicar que áreas potencialmente contaminadas.

O mesmo parâmetro, mas definido para o estado de Minas Gerais [11], tem como limite o valor de $8 \mathrm{mg} \cdot \mathrm{kg}^{-1}$ (Tabela 2), mais que o dobro daquele do estado de São Paulo, ainda assim a área estudada apresentou valores superiores. Mesmo assim, as concentrações de As de muitas das amostras de solos pesquisadas estão acima dos padrões estabelecidos pelo VRQ, portanto, indicando possível contaminação.

Defargeet al. [20] mostraram que as avaliações regulatórias atuais dos herbicidas mais utilizados no mundo são incorretas, e que estes contêm componentes, como o Arsênio, em sua composição. Este elemento é encontrado regularmente em níveis tóxicos em herbicidas à base de glifosato $\left(\mathrm{C}_{3} \mathrm{H}_{8} \mathrm{NO}_{5} \mathrm{P}\right)$, além de outros pesticidas, além do petróleo. Na cultura da soja é constante o uso de herbicidas contendo como princípio ativo o glifosato $\left(\mathrm{C}_{3} \mathrm{H}_{8} \mathrm{NO}_{5} \mathrm{P}\right)$. Seu uso comum se dá com a finalidade de combater a proliferação de plantas daninhas na lavoura, o que pode explicar os teores mais elevados de arsênio nas amostras [10-11] (Tabela 1; Tabela 2).

A CTC do PLINTOSSOLO PÉTRICO Concrecionário (Pedro Afonso) cultivado com feijão foi maior que $15 \mathrm{cmol} . \mathrm{dm}^{-3}$, tendo, por conseguinte, maior capacidade para trocar cátions e adsorver metais que o LATOSSOLO VERMELHO AMARELO Distrófico (Pedro Afonso), cuja CTC média situou-se entre $5-15 \mathrm{cmol} \mathrm{dm}^{-3}$ (Tabela 6) [14]. 


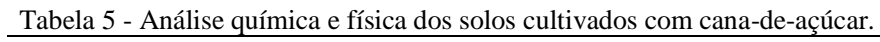

\begin{tabular}{|c|c|c|c|c|c|c|c|c|c|c|c|c|c|c|}
\hline pH & P (Mehlich) & $\mathbf{K}^{+}$ & $\mathrm{Ca}^{2+}+\mathrm{Mg}^{2+}$ & SB & $\mathbf{A l}^{3+}$ & $\mathbf{H}+\mathbf{A l}$ & CTC (pH 7,0) & $\mathbf{V}$ & $\mathbf{m}$ & M.O.S & $\mathbf{C}$ & Areia total & Silte & Argila \\
\hline \multirow[t]{2}{*}{$\mathbf{H}_{2} \mathbf{O}$} & \multicolumn{2}{|c|}{$\ldots . . . m g ~ \mathbf{~ d m}^{3} \ldots . . . . .}$. & & \multicolumn{4}{|c|}{ 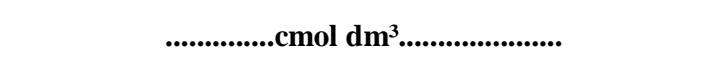 } & \multicolumn{4}{|c|}{ 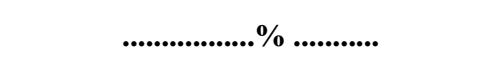 } & \multicolumn{3}{|c|}{$\ldots . . . . . . . \mathrm{g} \mathrm{kg}^{-1} \ldots . . . . . . . . . . . .}$. \\
\hline & \multicolumn{14}{|c|}{ LATOSSOLO VERMELHO AMARELO Distrófico 1 (Pedro Afonso) } \\
\hline \multirow[t]{2}{*}{5,47} & 5,78 & 50 & 5,13 & 5,26 & 0 & 9,65 & 14,91 & 35,26 & 0 & 2,65 & 1,538 & 702,4 & 34,4 & 263,2 \\
\hline & \multicolumn{14}{|c|}{ LATOSSOLO VERMELHO AMARELO Distrófico 2 (Pedro Afonso) } \\
\hline 4,82 & 3,5 & 40 & 2,27 & 2,37 & 0 & 11,78 & 14,15 & 16,76 & 0 & 2,16 & 1,25 & 482,4 & 41,6 & 476 \\
\hline
\end{tabular}

Tabela 6 - Análise química e física dos solos cultivados com feijão

\begin{tabular}{|c|c|c|c|c|c|c|c|c|c|c|c|c|c|c|}
\hline pH & P (Mehlich) & $\mathbf{K}^{+}$ & $\mathrm{Ca}^{2+} \mathrm{Mg}^{2+}$ & SB & $\mathbf{A l}^{3+}$ & $\mathbf{H}+\mathbf{A l}$ & $\begin{array}{l}\text { CTC (pH } \\
7,0)\end{array}$ & $\mathbf{V}$ & $\mathbf{m}$ & M.O.S & $\mathbf{C}$ & $\begin{array}{c}\text { Areia } \\
\text { total }\end{array}$ & Silte & Argila \\
\hline $\mathrm{H}_{2} \mathrm{O}$ & ......mg dn & $\ldots .$. & & & cmol d & .............. & & & .......... &..$\%$.......... & & \multicolumn{3}{|c|}{.............. k kg $^{-1} \ldots . . . . . . . . . . . . .}$. \\
\hline & \multicolumn{14}{|c|}{ LATOSSOLO VERMELHO AMARELO Distrófico (Pedro Afonso) } \\
\hline 5,68 & 9,62 & 90 & 4,33 & 4,56 & 0 & 6,63 & 11,19 & 40,74 & 0 & 2,87 & 1,667 & 769,6 & 34,4 & 196 \\
\hline \multicolumn{15}{|c|}{ PLINTOSSOLO PETRICO Concrecionário (Pedro Afonso) } \\
\hline 4,5 & 5,25 & 80 & 1,77 & 1,98 & 0,01 & 13,45 & 15,42 & 12,81 & 0,5 & 2,43 & 1,41 & 389,6 & 54,4 & 556 \\
\hline
\end{tabular}


O cádmio (Cd) não apresentou valores acima de 0,1 mg. $\mathrm{kg}^{-1}$ (Figura 1), por isso, não se aproximou do Valor de Investigação Agrícola (3,6 mg.kg $\left.{ }^{-1}\right)$ e nem mesmo do Valor de Prevenção (1,3 mg. $\mathrm{kg}^{-1}$ ) (Tabela 1) [10]. Não há, provavelmente, nenhum excesso desse elemento nos solos cultivados com soja. Contudo, vale ressaltar que as próprias características dos solos, podem apresentar propensão ao deslocamento do cádmio no solo, o que justifica a baixa concentração desse elemento nas camadas superficiais.

Estudos realizados por Kemerich et al. [21] corroboram essa característica do elemento. De acordo com a COPAM [11], o Valor de Referência de Qualidade para o cádmio $\left(<0,4 \mathrm{mg} . \mathrm{kg}^{-}\right.$ ${ }^{1}$ ) (Tabela 2) é mais restritivo que o designado pela CETESB [10] (Tabela 1). Mesmo assim, todos os solos sob o cultivo de soja não apresentaram teores elevados deste elemento.

$\mathrm{O}$ chumbo $(\mathrm{Pb})$ também não apresentou elevadas concentrações nas amostras pesquisadas, apresentando valores que atingiram até 3,2 mg. $\mathrm{kg}^{-1}$ (Figura 1), ou seja, muito abaixo do Valor de Prevenção (72 mg.kg ${ }^{-1}$ ) e de Investigação Agrícola (150 mg. $\mathrm{kg}^{-1}$ ) estabelecidos pela CETESB [10] (Tabela 1). Não há, portanto, indícios de possível contaminação.

Mesmo o VRQ para o chumbo estabelecido para o estado de São Paulo (CETESB [10]) (17 mg. $\left.\mathrm{kg}^{-1}\right)$ está muito acima dos valores encontrados nas amostras analisadas, levando a crer que não há riscos de contaminação dos solos por esse elemento. O VRQ para o estado de Minas Gerais (19,5 mg. $\left.\mathrm{kg}^{-1}\right)$ reflete a mesma lógica [11] (Tabela 2).

Os teores de cromo $(\mathrm{Cr})$ detectados nas amostras foram de até 47,2 mg. $\mathrm{kg}^{-1}$ (Figura 1), abaixo dos limites propostos pela CETESB [10] para Valor de Prevenção (75 $\left.\mathrm{mg} \mathrm{kg}^{-1}\right)$ e Investigação Agrícola (150 mg.kg ${ }^{-1}$ ) (Tabela 1).

Entretanto, segundo o VRQ estabelecido pela CETESB [10] (40 mg. $\left.\mathrm{kg}^{-1}\right)$, determinadas áreas sob o cultivo da soja apresentaram teores acima do limite estabelecido (Figura 1), indicando que deve haver uma preocupação quanto à alteração da qualidade do solo. É normal encontrar em áreas cultivadas, onde o revolvimento do solo ocorre apenas na camada de 0 a $20 \mathrm{~cm}$, maior concentração de $\mathrm{Cr}$ na camada superficial. No solo, o cromo pode ser encontrado, dependendo de sua especiação, na forma de complexos solúveis ou como partícula adsorvida em materiais argilosos, orgânicos ou óxidos de ferro. Estes compostos podem apresentar baixa solubilidade e reatividade, o que lhes confere características de baixa mobilidade [22].

A despeito do discutido acima, se considerado o VRQ indicado para o estado de Minas Gerais (75 mg. $\mathrm{kg}^{-1}$, ver Tabela 2) [11], os teores de cromo das amostras pesquisadas estão dentro dos limites estabelecidos. Esse aparente conflito entre os valores aponta para as particularidades de cada região e reforçam a necessidade de que o estado do Tocantins estabeleça seus próprios referenciais de monitoramento.

Quanto aos teores de mercúrio $(\mathrm{Hg})$ os valores máximos registrados nas amostras atingiram até $0,6 \mathrm{mg} \mathrm{kg}^{-1}$ (Figura 1), ou seja, acima do limite do Valor de Prevenção $\left(0,5 \mathrm{mg} . \mathrm{kg}^{-1}\right)$ indicado pelas normativas de ambos os estados e que serviram de apoio para a comparação destes resultados [10-11] (Tabelas 1 e 2). Esse resultado indica níveis de alerta desse elemento no solo, sendo necessário o monitoramento para uma possível intervenção. A contaminação do solo por metais pesados acontece quando a quantidade do metal exposto ao ambiente supera a capacidade na qual o solo consegue retê-lo [23]. Isso pode causar contaminação das águas de superfície e subsuperfície, levando também ao bem como sofrer efeito cumulativo dentro das cadeias alimentares.

Em contrapartida, os teores níquel (Ni) atingiram 0,9 mg. $\mathrm{kg}^{-1}$ (Figura 1), não apresentando risco de contaminação aos solos. Tendo em conta o VRQ estabelecido por CETESB [10] (igual a $13 \mathrm{mg} \mathrm{kg}^{-1}$ ) para o Estado de São Paulo e aquele estabelecido para o estado de Minas Gerais (21,5 mg. $\mathrm{kg}^{-1}$ ) [11](Tabela 2), os teores de níquel encontrados nas amostras parecem não apresentar potencial de contaminação. Um dos fatores que mais afeta o comportamento desse elemento no solo é o $\mathrm{pH}$, pois são variáveis inversamente proporcional [24].

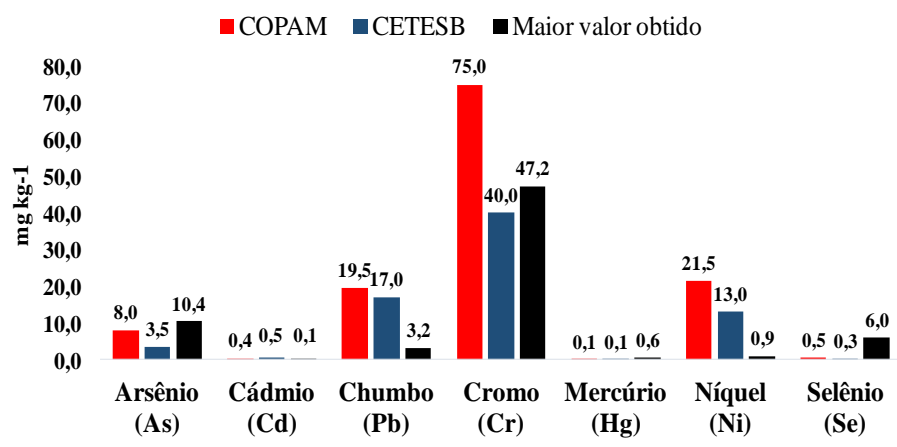

Figura 1 - Metais Pesados sob cultivo da soja, comparado com o Valores de Referência de Qualidade para os Estados de São Paulo (CETESB) e Minas Gerais (COPAM).

$\mathrm{O}$ selênio ( $\mathrm{Se}$ ) apresentou alguns valores que ultrapassaram o VRQ estabelecido para o Estado de São Paulo $\left(0,25 \mathrm{mg} \cdot \mathrm{kg}^{-1}\right)$ [10] (Tabela 1) e Minas Gerais (0,5 mg. $\left.\mathrm{kg}^{-1}\right)$ (COPAM, [11]) (Tabela 2), indicando a possibilidade de contaminação pelo selênio.

As fontes de metais pesados no solo agrícola, incluem os fertilizantes inorgânicos e orgânicos, a calagem, lodo de esgoto, águas de irrigação e pesticidas. Fertilizantes fosfatados têm níveis variáveis de cádmio, cromo, níquel, chumbo e zinco em função das suas fontes [25]. Isso é resultado da ocorrência natural 
de vários metais pesados em rochas fosfáticas, que não são eliminados no processo de manufatura.

\subsubsection{Teores de metais pesados em solos cultivados com milho}

Nos solos pesquisados e que, no momento da coleta, estavam cobertos pela cultura do milho, o arsênio atingiu valores de até $8,1 \mathrm{mg} \mathrm{kg}^{-1}$ (Figura 2). Este teor é superior ao do VRQ proposto para o estado de São Paulo (3,5 mg. $\mathrm{kg}^{-1}$ ) (Tabela 1) [10], embora seja praticamente idêntico àquele estabelecido para o estado de Minas Gerais (8 mg. $\left.\mathrm{kg}^{-1}\right)$ (Tabela 2) [11]. Isso parece indicar que apresentam-se inclinados à contaminação por esse elemento.

Quanto ao cádmio nenhuma amostra apresentou valor superior ao Valor de Prevenção (1,3 mg. $\mathrm{kg}^{-1}$ ) (Tabela 1). Com isso, todas as amostras também apresentaram valores dentro do limite estabelecido para Valor de Referência de Qualidade de acordo com a CETESB (0,5 mg. $\left.\mathrm{kg}^{-1}\right)$ (Tabela 1$)$ e COPAM $(0,4$ mg. $\mathrm{kg}^{-1}$ ) (Tabela 2).

Nestas mesmas amostras de solo, os níveis de chumbo não ultrapassaram 4,3 $\mathrm{mg} \mathrm{kg}^{-1}$ (Figura 2), muito abaixo do VRQ estabelecido pela COPAM [11] e CETESB [10] (17 $\mathrm{mg} \mathrm{kg}^{-1} \mathrm{e}$ $19,5 \mathrm{mg} \mathrm{kg}^{-1}$, respectivamente). Logo, os teores obtidos nas amostras indicam não haver risco de contaminação por esse elemento.

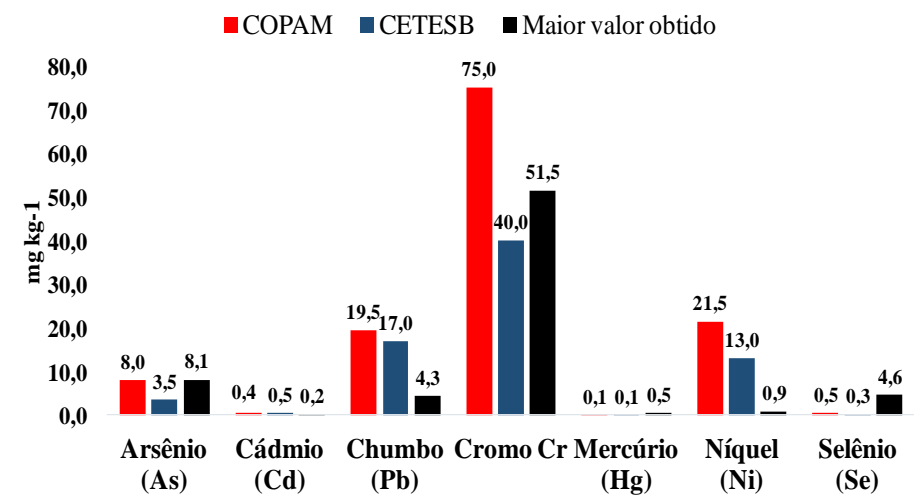

Figura2 - Metais Pesados sob cultivo do milho, comparado com o Valores de Referência de Qualidade para os Estados de São Paulo (CETESB) e Minas Gerais (COPAM)

As concentrações de cádmio e chumbo, nas amostras de solos sob cultivo de milho, foram superiores aos níveis encontrados nos solos cultivados com soja, o que provavelmente é o resultado do emprego de adubação fosfatada na cultura. Pesquisas comprovam um aumento desses elementos nesse tipo de adubação na cultura do milho. Silva [26] relata que os fertilizantes fosfatados apresentam em sua composição uma diversidade de metais. A alta solubilidade desses produtos pode promover absorção de metais por plantas, como mostram os trabalhos de Chien et al. [27], os quais observaram acumulação de cádmio na parte aérea de plantas de soja e milho, como consequência da aplicação de superfosfatos solúveis.

Para o cromo não foram identificadas concentrações acima dos Valores de Prevenção (75 mg. $\mathrm{kg}^{-1}$ ) e Investigação Agrícola (150 mg. $\mathrm{kg}^{-1}$ ) estabelecidos pela CETESB [10] (Tabela 1). Entretanto, o elemento chegou a concentrações de até 51,5 mg. $\mathrm{kg}^{-1}$ (Figura 2), acima do VRQ adotado como padrão pela CETESB [10] (40 mg. $\mathrm{kg}^{-1}$ ) (Tabela 1). Portanto, este elemento pode apresentar potencial de contaminação na área estudada.

O teor de mercúrio nas amostras (máximo de $0,5 \mathrm{mg} . \mathrm{kg}$ ${ }^{1}$, ver Figura 2) foi curiosamente idêntico ao Valor de Prevenção $\left(0,5 \mathrm{mg} \cdot \mathrm{kg}^{-1}\right)$ (Tabela 1$)$, mas muito acima do VRQ $\left(0,05 \mathrm{mg} \cdot \mathrm{kg}^{-}\right.$ $\left.{ }^{1}\right)$ estabelecido pela CETESB [10] e o COPAM [11] (Tabelas $1 \mathrm{e}$ 2), o que pode ser indício de algum nível de contaminação dos solos.

O níquel não apresentou valores expressivos, sempre abaixo dos Valores de Prevenção (30 mg. $\mathrm{kg}^{-1}$ ) e Valor de Investigação Agrícola (190 mg. $\mathrm{kg}^{-1}$ ) [10]; (Tabela 1). Da mesma forma, os teores nestas amostras foram menores do que aqueles do VRQ estabelecidos pela CETESB [10] (13 mg.kg-1) (Tabela 1) e pelo COPAM [11] (21,5 mg. $\left.\mathrm{kg}^{-1}\right)$ (Tabela 2).

O selênio, foi encontrado em concentrações que chegaram até 4,6 mg.kg-1 (Figura 2) e que, apesar de aparentemente não representar um valor alto, está acima do Valor de Prevenção (1,2 mg. $\mathrm{kg}^{-1}$ ) [10], e muito acima do Valor de Referência de Qualidade (0,25 mg. $\left.\mathrm{kg}^{-1}\right)$ (Tabela 1) [10]. O mesmo pode ser dito quando comparado estes números com aqueles determinados pela COPAM. Esse resultado indica que esses solos encontram-se em um regime de alta concentração de selênio, podendo representar riscos de contaminação de solo e água.

\subsubsection{Teores de metais pesados em solos cultivados com} cana-de-açúcar

Somente dois pontos amostrais foram escolhidos em solos sob cultivo de cana-de-açúcar e somente duas amostras foram tomadas. Nestas, quase todos os elementos apresentaram concentrações dentro dos padrões exigidos pela CETESB [10], além de estarem de acordo com ao Valores de Prevenções estabelecidos (Tabela 4) e dentro dos limites dos Valores de Referência de Qualidade [10-11].

Os teores de mercúrio (0,4 mg. $\mathrm{kg}^{-1}$; Figura 3), no entanto, alcançaram valor próximo ao de Prevenção (0,5 mg. $\left.\mathrm{kg}^{-1}\right)$ (Tabela 1), e superior ao de Referência de Qualidade [10-11], que é de $0,05 \mathrm{mg} \cdot \mathrm{kg}^{-1}$, sujeitando-o à interpretação de que existe um grande risco de estar contaminado por mercúrio. 


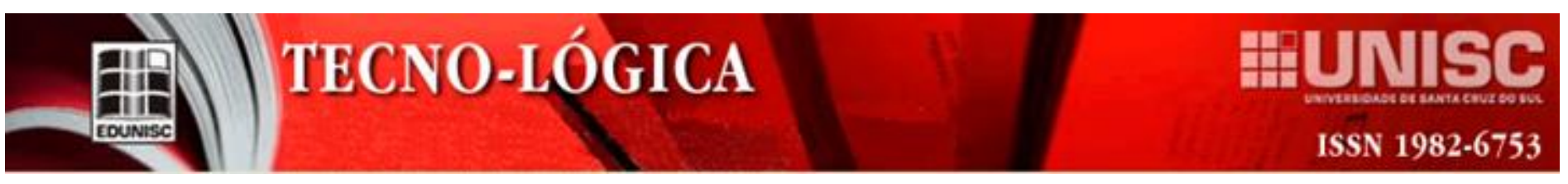

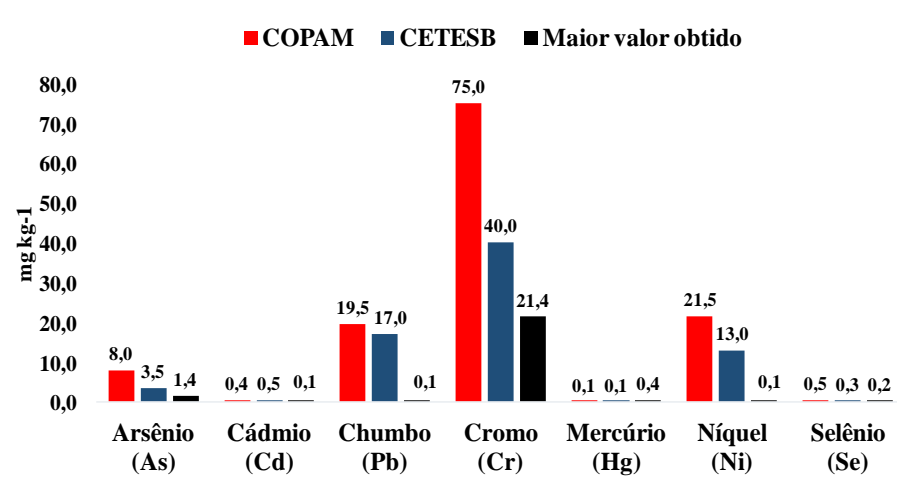

Figura 3 - Metais Pesados sob cultivo da cana-de-açúcar, comparado com o Valores de Referência de Qualidade para os Estados de São Paulo (CETESB) e Minas Gerais (COPAM).

É importante ressaltar que o maior valor para este elemento foi encontrado no município de Pedro Afonso, onde já havia sido relatado altas concentrações desse elemento no solo, segundo levantamento realizado pela Companhia de Pesquisa de Recursos Minerais (CPRM). Atividades de garimpo na região, mais precisamente na confluência do Rio do Sono com o Rio Tocantins, devem ser tidas como causas prováveis [28]. Embora desativadas, atividades desta natureza deixam resíduos que podem permanecer no solo por algum tempo.

O mercúrio é utilizado na separação de partículas finas de ouro, através da amalgamação e posterior separação gravimétrica. $\mathrm{O}$ amálgama separado é queimado, geralmente a céu aberto, liberando grandes quantidades de mercúrio para a atmosfera. Durante o processo, quantidades variáveis de mercúrio são perdidas na forma metálica para rios e solos.

\subsubsection{Teores de metais pesados em solos cultivados com feijão}

As amostragens em solos cultivados com feijão foram realizadas apenas em dois locais. Segundo os resultados analíticos, o arsênio não apresentou valor superior ao Valor de Prevenção (15 mg. $\left.\mathrm{kg}^{-1}\right)$ [10] (Tabela 1). No entanto, em uma das áreas seu teor foi superior ao Valor de Referência de Qualidade (3,5 mg. $\mathrm{kg}^{-1}$ ) indicado por CETESB [10] (Figura 4). Esta conclusão, entretanto, é contradita mediante comparação com o Valor de Referência estabelecido pelo COPAM [11] (8 mg. $\left.\mathrm{kg}^{-1}\right)$.

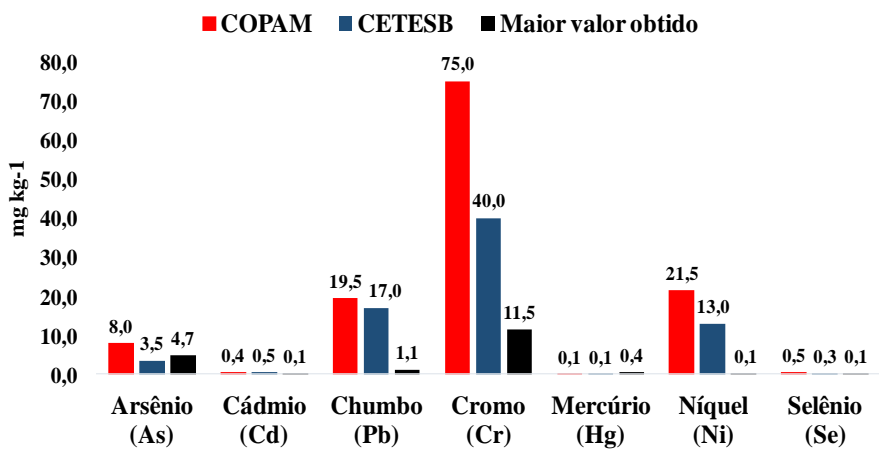

Figura 4 - Metais Pesados sob cultivo do feijão comparado com o Valores de Referência de Qualidade para os Estados de São Paulo (CETESB) e Minas Gerais (COPAM).

A concentração de mercúrio $(\mathrm{Hg})$ nos solos $(0,4 \mathrm{mg} \cdot \mathrm{kg}-$ $\left.{ }^{1}\right)$ foi bastante próxima ao Valor de Prevenção $\left(0,5 \mathrm{mg} \cdot \mathrm{kg}^{-1}\right)$ [10] (Tabela 1), e superior ao VRQ $\left(0,05 \mathrm{mg} \cdot \mathrm{kg}^{-1}\right.$; Figura 4$)$ de ambos os institutos tomados como referência [10-11]. Isso leva a crer que há necessidade de monitoramento.

Nota-se que nas áreas investigadas, os teores de mercúrio apresentados foram superiores em todas as culturas (soja, milho, cana-de-açúcar e feijão) quando comparados aos Valores de Referência de Qualidade [10-11], obtendo valor virtualmente idêntico ao Valor de Prevenção [10]. Isso acontece provavelmente devido ao uso excessivo de produtos químicos, como fertilizantes (principalmente os fosfatados).

Compostos à base de arsênio, selênio, chumbo e mercúrio são comuns dentre na formulação de produtos que caracterizaram a primeira geração de pesticidas. Desde os anos de 1980, o consumo industrial de mercúrio vem caindo substancialmente. Este fenômeno é resultado direto de uma legislação de controle mais eficiente, que resultou no banimento do uso de mercúrio em certos setores como nos defensivos agrícolas [29].

\section{Conclusões}

O mercúrio foi o elemento que mais frequentemente foi detectado nos solos estudados sob diferentes culturas, apresentando valores superiores aos níveis de Referência de Qualidade estabelecidos pelos estados de São Paulo e Minas Gerais.

Pode-se constatar que as maiores concentrações de metais pesados foram encontradas nas amostras de solos coletadas sob o cultivo da soja e de milho, e os elementos mais recorrentes foram os metais arsênio, mercúrio e o selênio. 
Nenhuma área obteve valor igual ou maior ao Valor de Investigação Agrícola (VI), o que poderia indicar o uso inapropriado ou inadequado do solo para atividades agrícolas.

As diferenças encontradas entre os Valores de Referência de Qualidade (VRQ) do estado de São Paulo e do estado de Minas Gerais, ressalta a importância e elevada urgência de que cada estado estabeleça seus valores orientadores.

\section{HEAVY METAL CONTAMINATION IN AGRICULTURAL AREAS IN TOCANTINS STATE}

ABSTRACT: The present work consisted in the collection of soils samples for the analysis and description of heavy metals values in agricultural areas of some municipalities in the state of Tocantins. The purpose is to make comparisons with the Reference Values established for the states of São Paulo (SP) and Minas Gerais (MG), once, there is no reference values do exist for the state of Tocantins. Soil samples were collected from 27 agricultural areas, and submitted to the EPA3051 digestion method, proposed by the Environmental Protection Agency (EPA). The soils samples showed different levels of heavy metal concentration, with arsenic (As), mercury $(\mathrm{Hg})$ and selenium (Se) being more constant. There has been no levels of heavy metals close to the Agricultural Research Values, only a few elements exceeded the limit of Prevention Values and the Quality Reference Values (QRV) proposed for the states of SP, as well as for the QRV established for the areas in the state of MG. These concentrations, however, pose no risk for soils contamination.

Keywords: CONAMA 420. Quality reference. Environment impact. Legislation.

\section{Referências}

[1] BRASIL, Conselho Nacional do Meio Ambiente - CONAMA. Resolução n. 420/2009, de 28 de dezembro de 2009. Dispõe sobre critérios e valores orientadores de qualidade do solo quanto à presença de substâncias químicas e estabelece diretrizes para o gerenciamento ambiental de áreas contaminadas por essas substâncias em decorrência de atividades antrópicas.2009. Diário Oficial da União, Brasília. 30 dec. 2009 Disponível em: http://www.mma.gov.br/port/conama/legiabre.cfm?codlegi=620. Acesso em 04 ago. 2019.

[2] COBBINA, S.J., CHEN, Y., ZHOU, Z., WU, X., ZHAO, T., ZHANG, Z., FENG, W., WANG, W., LI, Q., WU, X. \& YANG, L. Toxicity assessment due to sub-chronic exposure to individual and mixtures of four toxic heavy metals. Journal of Hazardous Materials. v. 294, p. 109-120, 2015.

[3] ALLOWAY, B.J. Heavy metals in soils. London: Blackie Academic and Professional, 1995. 368 p.

[4] ARAÚJO-MOURA, A.A.C., CAFFARO FILHO, R.A. Panorama do gerenciamento de áreas contaminadas no Brasil após a resolução CONAMA 420/09. Revista Água e meio ambiente subterrâneo - ABAS. v.29, n. 2, p. $202-$ $212,2015$.
[5] PEDROTTI, A. Sergipe estabelece os VRQs de seus solos para cumprimento à legislação ambiental. [Nossa Senhora da Glória -SE]: Universidade Federal do Sergipe, 2019. Disponível em: http://www.ufs.br/conteudo/63432-sergipeestabelece-os-vrqs-de-seus-solos-para-cumprimento-a-legislacao-ambiental. Acesso em: 16 out. 2019.

[6] CETESB - Companhia de Tecnologia de Saneamento Ambiental. Decisão de diretoria $\mathrm{n}^{\circ}$ 195-2005, de 23 de novembro de 2005. Dispõe sobre a aprovação dos Valores Orientadores para Solos e Águas Subterrâneas no Estado de São Paulo 2005, em substituição aos Valores Orientadores de 2001, e dá outras providências. São Paulo, SP. 2005. Disponível em: https://cetesb.sp.gov.br/solo/wp-

content/uploads/sites/18/2014/12/tabela_valores_2005.pdf. Acesso em: 04 ago. 2019 .

[7] SiBCS- Sistema Brasileiro de Classificação de Solos. 5. ed. Brasília: Embrapa. 2018, 356 p.

[8] EMBRAPA - Empresa Brasileira de Pesquisa Agropecuária. Centro Nacional de Pesquisas de Solos. Manual de métodos de análises de solos. 3.ed. Rio de Janeiro: Embrapa Solos. 2017, 577 p.

[9] USEPA - United States Environmental Protection Agency. Method 3051a: Microwave assisted acid digestion of sediments, sludges, soils, and oils. 1998. Washington, DC. Revision, 2007. 30p. Disponível em: https://www.epa.gov/sites/production/files/2015-12/documents/3051a.pdf. Acesso em: 02 abr. 2018.

[10] CETESB - Companhia de Tecnologia de Saneamento Ambiental. Decisão de diretoria $n^{\circ}$ 256/2016/e, de 22 de novembro de 2016. Dispõe sobre a aprovação dos "Valores Orientadores para Solos e Águas Subterrâneas no Estado de São Paulo - 2016" e dá outras providências. São Paulo, SP. 2016. Disponível em: https://www.cetesb.sp.gov.br/wp-content/uploads/2014/12/DD-256-2016-E-

Valores-Orientadores-Dioxinas-e-Furanos-2016-Intranet.pdf. Acesso em: 04 ago. 2019 .

[11] COPAM - Conselho Estadual de Política Ambiental. Deliberação Normativa COPAM n 166, de 29 de junho de 2011. Altera o Anexo I da Deliberação Normativa Conjunta COPAM CERH n 2 de 6 de setembro de 2010, estabelecendo os Valores de Referência de Qualidade dos Solos. Belo Horizonte, MG. 2011. Disponível em http://www.siam.mg.gov.br/sla/download.pdf?idNorma=18414. Acesso em: 04 ago. 2019.

[12] DALFIOR, B. M.; RORIZ, L. D.; F. JÚNIOR, R.; FREITAS, A. C.; SILVA, H. E.; CARNEIRO, M. T. W.D.; LICINIO, M. V. V. J.; BRANDÃO, G. P. Avaliação dos teores de $\mathrm{Pb}, \mathrm{Cd}, \mathrm{Sn}, \mathrm{Co}, \mathrm{Hg}$, Mo e As em solos da península Fildes - Antártica. Química Nova, São Paulo, v. 39, n. 8, p. 893-900, 2016. Disponível em: http://www.scielo.br/scielo.php?script=sci_arttext\&pid=S010040422016000800893\&lng=en\&nrm=iso. Acessoem: 16 out. 2019.

[13] SPOSITO, G. The chemistry of soils. 2ed. New York: Oxford University Press, 2008, 342 p.

[14] EMBRAPA - Empresa Brasileira de Pesquisa Agropecuária. Guia Prático para Interpretação de Resultados de Análises de Solo. $1^{\circ}$ ed. 13 p. Documentos 206 / Embrapa Tabuleiros Costeiros. Aracaju, SE: Embrapa Tabuleiros Costeiros, 2015, 13 p.

[15] DUMAT, C.; QUENEA, K.; BERMOND, A; TOINEN, S.; BENEDETTI, M. F. Study of the trace metal ion influence on the turnover of soil organic matter in cultivated contaminated soils. Environmental Pollution, Amsterdam, v.142, n. 3, p.521-529, 2006

[16] OLIVEIRA, F. C.; MATTIAZZO, M. E.; MARCIANO, C. R.; ABREU JUNIOR, C. H. Alterações em atributos químicos de um Latossolo pela aplicação de composto de lixo urbano. PesquisaAgropecuáriaBrasileira, Brasília, v. 37, n. 4, p. 529-538, 2002. 


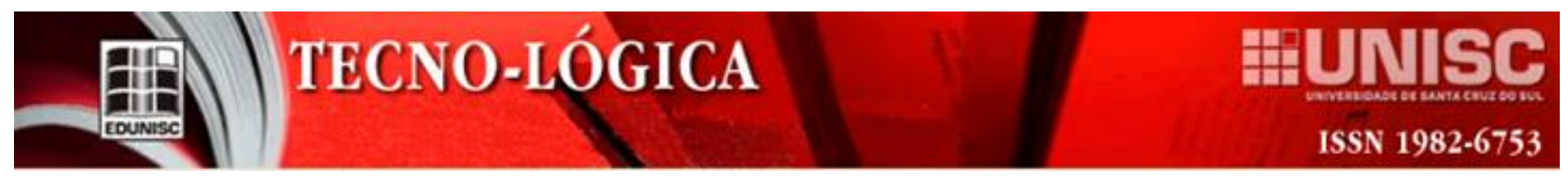

[17] GALÁN, E. The role of clay minerals in removing and immobilising heavy metals from contaminated soils. In: Proceedings of the Latin American Clay Conference, 1st., 2000. Funchal. Anais [...] Portugal, 2000. p. 351-361.

[18] STEVENSON, F.J. HumusChemistry: genesis, composition, reactions. 2. ed. New York: John Wiley, 1994, 496 p.

[19] SILVEIRA, M. L. A.; ALLEONI, L. R. F.; GUILHERME, L. R. G. Biosolids and heavy metal in soils. Scientia Agricola, v.60, n. 4, p.793-806, 2003.

[20] DEFARGE, N.; DE VENDÔMOIS, J. S; SÉRALINI, G. E. Toxicity of formulants and heavy metals in glyphosate-based herbicides and other pesticides. Toxicology Reports. v. 5, p. 156-163, 2018. doi: 10.1016/j.toxrep.2017.12.025

[21] KEMERICH, P. D. C.; FLORES, C. E. B.; BORBA, W. F. de; FLORES, B. A.; FILHO, L. L. V. D.; BARROS, G.; GERHARDT, A. E.; RODRIGUES, A. C. Variação espacial das concentrações de cádmio e manganês em solo ocupado por aterro sanitário. Revista Eletrônica em Gestão, Educação e Tecnologia Ambiental, v. 17, n. 17, p. 3336-3345, 2014. Disponível em: https://periodicos.ufsm.br/reget/article/view/10663/pdf. Acesso em: 15 out. 2016.

[22] HELENE, L. P. I. Diagnóstico ambiental de solo contaminado por cromo de curtume em Motuca (SP) por métodos geofísicos. 2016. 74 f. Dissertação (Mestrado em Geociências e Meio Ambiente) - Universidade Estadual Paulista, Instituto de Geociências e Ciências Exatas Rio Claro, São Paulo. 2016.

[23] SIQUEIRA, G.; APRILE, F. Avaliação de risco ambiental por contaminação metálica e material orgânico em sedimentos da bacia do Rio Aurá, Região Metropolitana de Belém - PA. Acta Amazonica, v. 43, p. 53-64, 2013. Disponível em: http://www.scielo.br/pdf/aa/v43n1/v43n1a07.pdf. Acesso em: 20 ago. 2019.

[24] REIS, A. R.; RODAK, B.W.; PUTTI, F.F.; MORAES, M.F. Papel fisiológico do níquel: essencialidade e toxidez em plantas. Informações Agronômicas, IPNI, Piracicaba, v.147, p. 10-24, 2014.

[25] SOUZA, K. R.; MORASSUTI, C. Y.; DE DEUS, W. B. Poluição do ambiente por metais pesados e utilização de vegetais como bioindicadores. Acta BiomedicaBrasiliensia, v.9, n. 3, p. 95-106, 2018.

[26] SILVA, J. T. A. Fator de enriquecimento (FE) de cádmio (Cd) em solos sob agroecossistema de pipericultura no nordeste do Pará. 2017. 42 f. Trabalho de Conclusão de Curso (Bacharelado em Ciências Biológicas) - Universidade Federal Rural da Amazônia, Capanema. 2017.

[27] CHIEN, S.H.; CARMONA, G.; PROCHNOW, L.I.; AUSTIN, E.R. Cadmium availability from granulated and bulk-blended phosphate-potassium fertilizers.Journalof Environmental Quality, v. 32, p. 1911-1914, 2003.

[28] COSTA, D. T.; MOREIRA, M. R.; RODRIGUES, R. M. M.; CAMARGO, S. T.; FREITAS, U. R. Avaliação e diretrizes para o setor mineral do estado do Tocantins. Palmas, TO, SEPLAN, 2007, 92 p.

[29] OLIVEIRA, D. S.; NUNES, C. V. B.; DE JESUS, L.; LOIOLA, S. A. S.; SANTOS, A. C. A. Impactos do mercúrio no meio ambiente e na saúde. In:Seminário Pesquisar, 6, 2016, Aparecida de Goiânia. Anais [...]. Aparecida de Goiânia: Faculdade Alfredo Nasser, 2016. Disponível em: http://www.faculdadealfredonasser.edu.br/files/Pesquisar_5/21-11-201621.40.55.pdf. Acesso em: 16 out. 2019. 